\title{
Die Folgen der Tschernobyl-Katastrophe
}

\author{
Berichte von Ärzten, die vor Ort Erfahrungen über die Tschernobyl-Opfer gesammelt \\ haben, sind oftmals auf Russisch oder Ukrainisch verfasst. Die New York Academy of \\ Sciences hat sie in einem Sonderheft zusammengefasst und ins Englische übertragen, \\ damit sie auch westlichen Medizinern zugänglich sind.
}

1 Yablokov AV et al. Chernobyl. Consequences of the Catastrophe for people and the environment. Annals of the New York Academy of Sciences. Vol. 1181 (2009).

Korrespondenz:

Prof. Dr. med. Jürg Ulrich Grellingerstrasse 65 CH-4052 Basel

juerg.ulrich@unibas.ch
Befürworter der Nuklearenergie behaupten, die Folgen des Unfalles von Tschernobyl seien im Grunde geringfügig gewesen, hätten im wesentlichen den Tod einiger Dutzend Mitarbeiter des Kraftwerks verursacht und wenige hundert Fälle von operablem Schilddrüsenkarzinom bei Kindern verursacht. Das sei ein zumutbares Opfer, wenn man den ungeheuren Zuwachs an Energie bedenke, den die Kernkraftwerke mit sich brächten. Es sei geringfügiger als etwa der Tod bei Unfällen in Kohlegruben, von denen man fast jedes Jahr in den Medien hören könne.

Wer aber Gelegenheit hatte, mit Geschädigten der Katastrophe von Tschernobyl zu sprechen, kann sich des Eindrucks nicht erwehren, dass hier Menschen infolge der äusseren Bestrahlung oder der im Körper aufgenommenen radioaktiven Isotope in ihrer körperlichen und geistigen Gesundheit zutiefst geschädigt wurden. Ärzte, die nach dem 26. April 1986 in mit radioaktiven Substanzen verseuchten Gebieten arbeiteten, schliessen sich meist dieser schwerwiegenden Beurteilung an. In den am meisten betroffenen Gebieten teilten solche Ärzte ihre Erfahrungen in Hunderten von wissenschaftlichen Arbeiten mit.

Da die Mehrzahl dieser Mitteilungen in russischer oder ukrainischer Sprache verfasst ist, werden sie nur in geringem Ausmasse von den Ärzten Westeuropas und Amerikas wahrgenommen. Es ist deshalb ein besonderes Verdienst der New York Academy of Sciences, dass sie in einem Sonderheft eine Zusammenstellung solcher Arbeiten publiziert hat [1]. Die russischen und weissrussischen Autoren dieses Hefts sind auch im Westen wegen ihrer Fachkenntnis hoch angesehen. A. V. Yablokov ist Biologe, Mitglied der Russischen Akademie der Wissenschaften, V. B. Nesterenko hat in Minsk das wissenschaftliche Institut «Biorad» aufgebaut, das die Folgen des Ereignisses vom 26. April 1986 registriert. Als einer der ersten flog er im Helikopter über den Unglücksreaktor. Er starb im Herbst 2008 aus dem Autor unbekannten Gründen. A. V. Nesterenko ist sein Sohn, der jetzt das Minsker Institut leitet und dessen Tradition weiter pflegt.

Die geographische Verteilung der radioaktiven Belastung lag zu 57\% ausserhalb der damaligen Sowjetunion - unter anderem in Deutschland und der Schweiz. Im Ganzen leben über 400 Millionen Menschen in Gebieten, deren Verstrahlung zwischen dem 26. April 1986 und Ende Juli 1986 höher war als 0,11 Curie pro Quadratkilometer (4 kilo-Becquerel pro

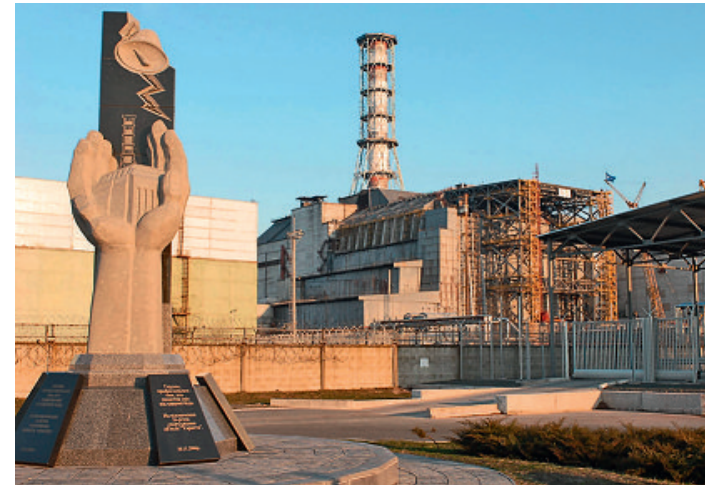

Monument beim Kraftwerk Tschernobyl zum Gedenken an den Super-GAU am 26. April 1986.

Quadratmeter). Zurzeit leben immer noch über fünf Millionen Menschen in stark verstrahlten Gebieten.

$\mathrm{Zu}$ den verursachten Gesundheitsschäden gehören die Chromosomenanomalien, insbesondere das vermehrte Vorkommen der Trisomie 21, das in Westberlin und Weissrussland beobachtet wurde und noch heute über dem früheren Niveau liegt. Auch entstellende, sonst kaum je beobachtete Missbildungen der Extremitäten traten auf. Als neue Krankheit ist eine Degeneration des Myokards bei Kindern zu nennen, das unter dem Einfluss von pectinhaltigen Medikamenten und nicht kontaminierter Nahrung reversibel scheint.

An Tumoren sind neben Schilddrüsenkarzinomen in allen Altersstufen, Leukämien und andere maligne Veränderungen des blutbildenden Systems, vermehrte Brust- und Lungenkarzinome zu nennen. Im Gegensatz zu Schätzungen offizieller internationaler Agenturen von 9000 bis 28000 tödlichen Geschwülsten schätzt Yablokov ihre Zahl allein in Europa auf 212000 bis 245000 . Er vermutet, dass ihre Tschernobyl-bedingte Vermehrung noch über Generationen anhalten wird.

Es ist wichtig, diese Bedrohung zu sehen und ihre Realisierung zu vermeiden, indem man die Einnahme kontaminierter Nahrung, das Pflügen kontaminierter Böden und das Verbrennen von Pflanzenresten vermeidet, in denen radioaktive Isotope angereichert sind. Missbildungen an Kleinlebewesen und abnormes Gefieder von Vögeln sind Hinweise auf Mutationen, die meist Folgen strahlenaktiver Isotope sind.

Wer das leider sehr teure Heft aufmerksam liest, gewinnt die Überzeugung, dass die Menschheit noch während vieler Jahre mit den Folgen von Tschernobyl zu ringen haben wird. 